\title{
Le Conseil des Etats, chambre de réflexion?
}

Que le système politique suisse vive une période de crise est une évidence - du Conseil fédéral à chacun de nos Parlements, des questions de fond se posent sur la manière de continuer à diriger le pays et les cantons de façon adéquate, dans un siècle qui est maintenant sérieusement entamé ... et où les bonnes habitudes n'en sont manifestement plus. Composition des exécutifs, place des partis, rôle des cantons et du Parlement, gestion des conflits d'intérêt, répartition des compétences, tout est mis en question, et tout est d'ailleurs aussitôt objet de jeux de pouvoir.

Nous y sommes confrontés comme tout citoyen, comme toute citoyenne, mais aussi parce que l'action politique autour du système de santé est désespérément marquée par ces tensions et par les blocages qui en résultent.

Il doit cependant être dit clairement ici que nous ne sommes pas décidés, comme corps médical, comme groupe professionnel, à payer les frais de cette crise qui ne nous concerne pas comme tels, ni à en subir indûment les contrecoups.

Autrement dit, concrètement, il n'est pas acceptable pour nous que les intérêts particuliers s'imposent face à la volonté populaire, ou que le pouvoir économique puisse prendre l'ascendant sur le pouvoir politique.

C'est pourtant ce qui s'est passé fin août lorsque la Commission de la santé du Conseil des Etats a froidement jeté aux orties la proposition de gestion de la démographie médicale qu'avec la CDS, c'est-à-dire avec les cantons, nous avions élaborée à sa demande pendant l'été.

Cette Commission de treize membres comprend cinq ou six députés qui sont étroitement liés aux grandes compagnies d'assurance de ce pays, et qui ont bien vite oublié que $70 \%$ de la population a rejeté il y a à peine trois mois toute idée de limiter le libre choix du médecin; ils ont donc une nouvelle fois mandaté l'administration fédérale pour qu'elle prépare une loi prévoyant la «liberté de contracter» pour les assureurs, sur la base d'un projet émanant de santésuisse.

Outre que l'option choisie par la Commission du Conseil des Etats est une vieille lune dont le côté passéiste est désespérant, il faut aussi noter qu'elle ne répond d'aucune façon au problème qui se pose en réalité maintenant, qui est celui de la suite de la «clause du besoin».

Sans règles prévues à l'expiration de cette dernière, à fin 2009, nous risquons le chaos dans notre système de santé, avec ce que cela suppose de mesures d'urgence mal réfléchies et de conséquences douloureuses.

Doit-on se demander si certains souhaitent ce chaos pour imposer leurs vues?

La FMH va, dans les semaines et les mois qui viennent, continuer à s'investir pour proposer des solutions adéquates aux problèmes qui nous attendent, pour tenter de contourner le blocage existant au Parlement, ... et pour refuser énergiquement les solutions boiteuses et les intrigues maladroites qui priveraient notre système de santé des perspectives d'avenir qui doivent être les siennes.

Nos contacts avec les partis et les parlementaires iront dans ce sens, avec essentiellement les options suivantes:

- assurer une démographie médicale souple et adaptée aux besoins, dans toutes les régions du pays;

- permettre à l'ensemble de la population un accès aux soins sans barrière financière inadéquate ou injuste, telle que l'entraînerait la «liberté de contracter»;

- préserver des conditions de travail et de vie correctes pour les médecins.

Et nous ne manquerons pas de faire appels à nos groupements régionaux et cantonaux, ainsi qu'aux sociétés de discipline, pour nous appuyer dans ces démarches lorsque cela sera nécessaire.

Dr Jacques de Haller, président de la FMH 